\title{
Historia de la carrera Ciencias Sociales de la UNAN-Managua
}

\section{Información de artículo:}

Recibido: 30/03/2021

Aprobado:30/05/2021

Palabras claves:

Ciencias Sociales, Historia, Curriculum, Educación.

\section{Key Words:}

Social Sciences, History, Curriculum, Education

\section{Resumen}

La historia de la carrera Ciencias Sociales de la Facultad de Educación e Idiomas de la UNAN-Managua hace un denso recorrido sobre un conjunto de hechos y vivencias de carácter académico, curricular y organizativo de la carrera entre los años de 1960 a la actualidad, también entremezcla en las entrañas de sus relatos aquellas particularidades sociopolíticos y culturales que de forma directa e indirecta incidieron en la construcción de los diversos planes de estudios de la Universidad y de la carrera Ciencias Sociales. Esta historia ha sido reconstruida a través de las memorias y relatos de personas claves que por medio de la metodología de la Historia Oral se lograron entrevistar, asimismo de la información obtenida mediante los análisis bibliográficos de documentos, actas, planes de estudios y cartas oficiales. Así, como consecuencia de este exhaustivo proceso investigativo, se coloca en manos del lector un artículo que caracteriza cada etapa de la carrera Ciencias Sociales con especial énfasis en el contexto social en que se desarrolla, las condiciones académicas y administrativas en que se situaban, las condiciones físicas en que se desarrollaba, los planes de estudios que comprendía, el personal docente con el que contaba, y otras características particulares que se recopilaron de cada momento histórico.

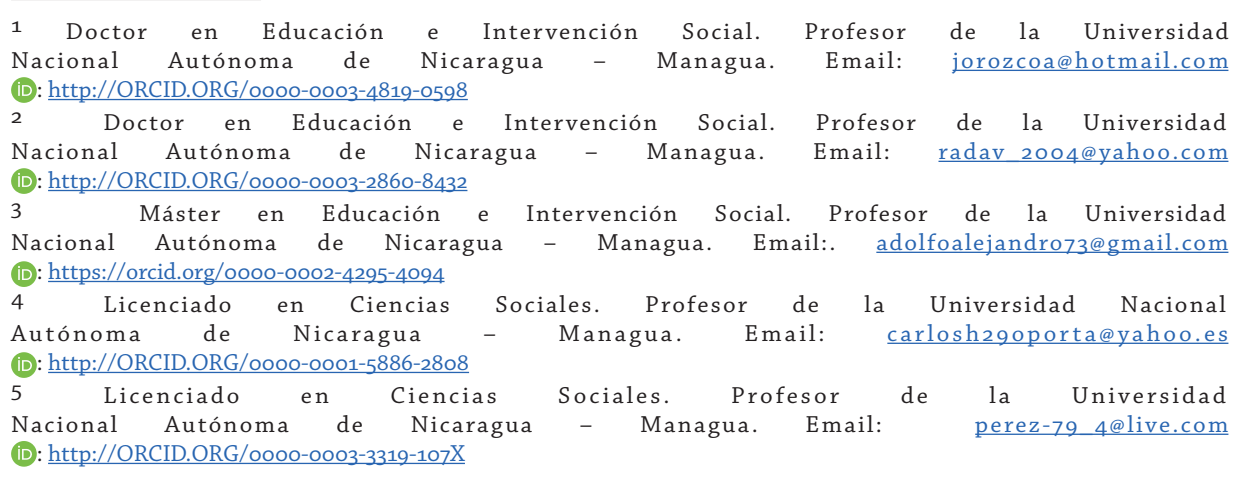




\section{History of the social science career of UNAN-Managua}

\section{Abstract}

The history of the Social Sciences career of the Faculty of Education and Languages of the UNAN-Managua makes a dense journey on a set of facts and experiences of academic, curricular and organizational character of the career between the years 1960 to the present, also intermingles in the bowels of their stories those sociopolitical and cultural particularities that directly and indirectly influenced the construction of the various curricula of the University and the Social Sciences career. This history has been reconstructed through the memories and stories of key people who were interviewed by means of the Oral History methodology, as well as the information obtained through the bibliographic analysis of documents, minutes, study plans and official letters. Thus, as a result of this exhaustive research process, the reader is provided with an article that characterizes each stage of the Social Sciences career with special emphasis on the social context in which it developed, the academic and administrative conditions in which it was located, the physical conditions in which it was developed, the curricula it included, the teaching staff it had, and other particular characteristics that were compiled for each historical moment.

\section{Introducción}

El presente artículo científico titulado Historia de la carrera de Ciencias Sociales de la Universidad Nacional Autónoma de Nicaragua (UNAN-Managua), tiene como propósito primordial conocer el origen y fundación de la carrera Ciencias Sociales de la Universidad Nacional Autónoma de Nicaragua, Managua (UNAN-Managua), así como indagar la caracterización histórica, el perfil de los egresados y las diferentes transformaciones curriculares que ha tenido la carrera desde su fundación hasta la actualidad.

El problema que conllevó a la realización de esta investigación es que en la actualidad no existe ningún trabajo a profundidad que narre y sistematice los hitos de la carrera de Ciencias Sociales de la UNAN-Managua, motivando así al colectivo de académicos de la carrera, a la realización de este artículo dando a conocer así a la sociedad y al estudiantado de la universidad como surgió esta carrera que es de gran importancia para la formación del futuro profesorado de Educación Secundaria.

Dicha investigación es de suma importancia para la UNAN-MANAGUA y la carrera, porque se recopila información y testimonios de vidas que narran la historia de la carrera Ciencias Sociales en orden cronológico abordando aquellos acontecimientos o cambios que son de relevancia, cabe destacar que la carrera Ciencias Sociales es una de las pocas que desde su apertura nunca ha sido cerrada, sino que ha estado activa continuamente en todas las épocas y contextos. 


\section{Literatura}

En este capítulo se definen algunos aspectos que darán mayor validez científica al presente artículo y que son necesarios para que los lectores estén claros de algunos términos que se narran en los diferentes testimonios de vida que nos brindaron los informantes sobre la carrera de Ciencias Sociales.

\section{- Perfil de la carrera ciencias sociales.}

De acuerdo a Morazán (2015) explica que el perfil de una carrera profesional se define como el "conjunto de rasgos, capacidades, habilidades, conocimientos y competencias que, aunados a ciertas actitudes, permiten que la persona sea acreditada legalmente por una institución educativa, a fin de que sea reconocida dentro de la sociedad como un profesional"

El perfil de la carrera Ciencias Sociales, es la enseñanza y el aprendizaje de las Ciencias Sociales en Institutos de Educación Secundaria y Centros de Educación Técnica del país, este profesional es capaz de comunicarse en diferentes contextos, mostrar respeto hacia la diversidad cultural de los pueblos y su ambiente, dominar de manera científica y metodológica la enseñanza y el aprendizaje de las ciencias sociales, asimismo, está preparado para aplicar el método científico en la realización de investigaciones de carácter socioeducativo, que den respuestas a los problemas inherentes a su campo profesional, posee habilidades para brindar acompañamiento pedagógico, asesoría escolar. Además, gestionar procesos administrativos vinculados con la educación, a fin de incidir en la mejora de la calidad educativa y fomentar valores que aporten a la formación ciudadana y al desarrollo del país.

\section{- Plan de Estudios curricular}

El plan de estudios y los programas curriculares son documentos guías que prescriben las finalidades, contenidos y acciones que son necesarios para llevar a cabo por parte del maestro y sus alumnos para desarrollar un currículum, (Casarini, 1999, p.8). Este es la síntesis instrumental mediante la cual se seleccionan, organizan y ordenan, para fines de enseñanza, todos los aspectos de una profesión que se considera social y culturalmente valiosos, profesionalmente eficientes.

El plan de estudios no es una estructura fija y sapiente que alberga el contenido organizado del aprendizaje. Se trata de un instrumento dinámico y refleja las metas, experiencias y competencias educativas a ser alcanzadas. Dado que la sociedad cambiará con el tiempo, en consecuencia, el plan de estudios cambiará y se desarrollará a medida que el programa se pone en marcha. 


\section{- Transformación curricular}

La transformación curricular es definida como las reformas y actualizaciones de los programas y planes de estudios realizando cambios en los enfoques, los paradigmas educativos, los métodos, contenidos y competencias que se quieren desarrollar en los estudiantes que se están preparando profesionalmente y que estos sean capaces de satisfacer las demandas del contexto y de la sociedad actual. Dichas transformaciones se realizan con el objetivo de mejorar la calidad educativa.

\section{Metodología de investigación}

La presente investigación se trabajó bajo el paradigma interpretativo, al respecto Fernández y Rivera, (2015, p.93) expresa que este paradigma permite tratar los fenómenos sociales e interpretar y comprender lo que ocurre en diferentes contextos humanos. Desde esta perspectiva, el investigador tiene libertad para "describir los sucesos que ocurren en la vida de un grupo, comprender y describir una situación determinada" (Abraham, 2015, p.120). Este paradigma es importante en la investigación educativa porque permite que el investigador asuma una visión interpretativa de la realidad, y pueda, de esta manera, comprender, interpretar y dar significada a la vida social o al fenómeno que está estudiando.

El enfoque que predomina en la investigación es el cualitativo. De acuerdo con Hernández Sampieri, Fernández Collado y Baptista Lucio (2014) este enfoque se fundamenta en una perspectiva interpretativa centrada en el entendimiento del significado de las acciones de seres vivos, para "obtener las perspectivas y puntos de vistas de los participantes [recuerdos, emociones y experiencias]" y recopilar "las vivencias de los participantes tal como fueron (o son) sentidas y experimentadas" (p.8).

En este sentido, la presente investigación es interpretativa con enfoque cualitativo ya que su principal objetivo fue reconstruir la historia de la carrera de Ciencias Sociales de la Facultad de Educación e Idiomas de la UNAN-Managua, a través de los testimonios y narraciones recopiladas de sus informantes por medio de las entrevistas realizadas.

El tipo de investigación es histórica, para Bernal (2010) la investigación histórica consiste en "estudiar y examinar los fenómenos, como producto de un determinado desarrollo, desde el punto de vista como han aparecido, evolucionado y llegado al estado actual" (p.110). Se pretende entender la sucesión de hechos, situaciones, fenómenos, procesos y eventos donde se involucran pensamientos, sentimientos, emociones e interacciones, a través de las vivencias contadas por quienes los experimentaron.

La metodología de la investigación hace referencia al método de historia oral. Al respecto Rodríguez, Luque y Navas (2014) expresan que la historia oral implica la narración de hechos y sucesos pasados que son expresados a viva voz, fomentado 
la recuperación de la memoria histórica a través de las vivencias, las experiencias y sensaciones vividas que son recogidas de manera escrita.

Por lo tanto, en el presente caso la metodología fue concebida como la forma y el camino que se trazó con el fin de lograr el objetivo de construir la historia de la carrera Ciencias Sociales de la Facultad de Educación e Idiomas de la UNAN-Managua, y para ello, el equipo de investigadores asumió los referentes teóricos de la metodología de la Historia oral.

\section{Historia de la carrera Ciencias Sociales de la UNAN-Managua}

\section{La carrera Ciencias Sociales hacía su sexagésimo primer año de fundación (1960 - 2021)}

La carrera Ciencias Sociales de la UNAN-Managua, en su devenir ha dejado un ramillete de hechos e hitos que componen memorias en las centenas de generaciones que han formado parte de su historia. En su recorrido histórico se visualizan cambios sustanciales suscitados principalmente por la incidencia de las nuevas realidades nacionales e internacionales que la historia ha venido marcando en los distintos momentos, trazando así un panorama histórico lleno de vaivenes de relieves pujantes, sombríos y complejos que han podido ser superados hasta la actualidad, a tal punto de ser la única carrera de la Universidad que ha sido ofertada ininterrumpidamente desde su fundación hasta la actualidad.

Así pues, la carrera Ciencias Sociales, en sus auges y en sus llanos ha sido tierra de nadie y codicia de todos, en su momento enajenada a otras áreas del conocimiento y nutrida por otros perfiles profesionales, pero sosteniendo inalterablemente su perfil profesional que paulatinamente ha podido ir estableciendo con mayor hondura a lo largo de los tiempos hasta imprimir un sello único de cientista sociales, siendo este la formación de profesionales con amplio dominio de los saberes de las distintas disciplinas sociales, el desarrollo de los conocimientos psicopedagógicos para el ejercicio de la docencia y el aprendizaje de lo socioeducativo con el propósito de aportar a la solución de problemáticas no solo educativas sino también sociales.

En la actualidad, la carrera Ciencias Sociales no dista de sus orígenes y de su historia, sus tiempos de bonanza, rezago y de altibajos la han conducido a replantear y redefinir su naturaleza y alcanzar una madurez académica más contundente. Actualmente es una carrera que pertenece al Departamento Multidisciplinario de la Facultad de Educación e Idiomas de la UNAN-Managua, y como todos los tiempos que le anteceden, sigue formando profesionales competentes para el desarrollo del país en los ámbitos educativos, investigativos y de trabajo social. 


\section{Los inicios de la carrera Ciencias Sociales (07 de julio de 1960)}

Los inicios de la carrera Ciencias Sociales se remontan hacia el o7 de julio de 1960 (Genet, 2020) cuando en ese entonces, lo que hoy es la UNAN-Managua, era apenas una extensión de la UNAN-León, y fue nombrada legalmente como Recinto Universitario Rubén Darío. En este Recinto se fundaron por primera vez en Managua las Facultades de Economía y de Ingeniería, y la Escuela de Ciencias de la Educación, esta última ofertaba las carreras de Letras, Matemática, Química-Biología y Ciencias Sociales, todas ellas con el enfoque de formación del profesorado para la Enseñanza Media y a nivel de Licenciatura, siendo su sede administrativa en la UNAN-León, por consiguiente, cabe puntualizar que la andadura de estas carreras se inicia en la UNAN-León.

Así pues, cuando la carrera Ciencias Sociales de apertura en Managua, esta da continuidad al Plan de Estudios diseñado por la UNAN-León el cual, de acuerdo a la revisión documental del Plan de Estudios de 1970 se encontró que los estudiantes cursaban un año de Estudios Generales, también llamada Año Básico, y luego cursaban tres años profesionalizantes, en suma, la carrera tenía una duración de cuatro años que equivalían a ocho semestres.

Asimismo, este Plan de Estudios graduaba a profesionales bajo la nomenclatura de Licenciado en Ciencias de la Educación mención Ciencias Sociales, siendo su distribución de carga académica por área la siguiente: El 46.5\% estaba conformado por asignaturas disciplinares que correspondían al área de Geografía, Historia, Antropología, Derecho, Filosofía, Sociología y Economía, el 32.5\% lo ocupaban las asignaturas básicas, entre ellas Biología I y II, Estudio de la Cultura I y II, Español I y II, Matemática Introductoria, Inglés General I, II y III, Francés General I, II y III, e Introducción a la Estadística; el 11.6\% correspondía a las asignaturas de corte psicopedagógico, apenas el $4.6 \%$ correspondía a dos asignaturas de naturaleza investigativa y solamente el $2.3 \%$ correspondía a una asignatura del área de didácticas especiales y de prácticas docentes respectivamente.

A inicios de 1980, siendo Nicaragua el epicentro de un estallido revolucionario que dio fin a la dictadura militar somocista, propiamente el 19 de julio de 1979, el Recinto Universitario Rubén Darío de Managua acoge una fuerte demanda estudiantil debido a que se asume una educación pública comprometida con el pueblo, y como consecuencia, todas las carreras experimentan un creciente auge que empujaron al Recinto a alcanzar su autonomía académica, orgánica, administrativa y financiera respecto a la UNAN-León, por medio del Decreto 1036 el 29 de abril de 1982, instituyéndose así, la UNAN-Managua (UNAN-Managua, 2019).

Como parte de estos cambios, a lo interno de la Universidad se comienza a reorganizar su estructura académica y administrativa y en 1980 surge la Facultad de 
Ciencias de la Educación, a la cual pertenecía la carrera de Ciencias Sociales, siempre con el enfoque de formación del profesorado a nivel de Licenciatura, con la particularidad que con la primera gran reforma curricular de la UNAN-Managua en el año 1982, ya una vez autónoma respecto a la UNAN-León, el Plan de Estudios de la carrera Ciencias Sociales contemplaba una bifurcación en la especialidad de Historia o Geografía a la altura IV año, de tal modo, que los estudiantes se graduaban bajo el título de: Licenciado(a) en Ciencias de la Educación en la especialidad de Geografía o bien, Licenciado(a) en Ciencias de la Educación en la especialidad de Historia.

En cuanto a las características curriculares del Plan de Estudios 1982 de la carrera Ciencias Sociales, es preciso señalar que este estaba más enfocado en el área científica de las distintas disciplinas sociales, por decir, filosofía, economía, historia, geografía, sociología etc., por ejemplo, de las 40 asignaturas que contenía el Plan de Estudios 82 en la especialidad de Geografía, el 52.5\% estaba conformado por asignaturas disciplinares que correspondían al área de Geografía, Historia, Filosofía y Economía, el 22.5\% lo ocupaban las asignaturas básicas, el 17.5\% las asignaturas de corte psicopedagógico, solamente el 2.5\% correspondía a una asignatura de la didáctica especial, y apenas el $5 \%$ correspondía a asignaturas de investigación.

Como consecuencia de este enfoque en las disciplinas científicas, los profesionales egresados carecían del perfil pedagógico con el cual debían de haber sido formados y, por ende, el Ministerio de Educación (MED) de Nicaragua hacía sentir la necesidad de disponer de profesionales con mayor perfil educativo para la enseñanza en Educación Secundaria. Esto conllevó a crear una comisión de carrera y a reestructurar el plan de estudio, incorporando un perfil con mayor énfasis en la docencia, lo cual logró concretarse con la transformación curricular de 1987.

En el año 86, casi a mediados como en el mes de junio por ahí se establece la comisión de carrera de Ciencias Sociales, ya centrado en un perfil que sin olvidar lo histórico, lo geográfico y otras ciencias sociales, se centraba fundamentalmente en la captación de recursos para la enseñanza media (Lobato, 2020).

\section{La Escuela de Ciencias Sociales (1983 - 1986)}

En los primeros años de la Revolución Popular Sandinista, se experimentó una creciente demanda en la carrera Ciencias Sociales, particularmente por el contexto sociohistórico de la época y por el perfil de la carrera, el cual se conjugaban perfectamente, sumado a esto, también cabe destacar la fuerte incidencia que tuvo la Cruzada Nacional de Alfabetización en 1980; esto conllevó a que en la carrera se acogiera estudiantes provenientes de las Milicias Populares Sandinistas, trabajadores del Estado, miembros de las estructuras partidarias comunitarias y profesionales del partido 
Frente Sandinista de Liberación Nacional en su mayoría ${ }^{6}$, sin embargo, también habían estudiantes que se habían integrado a la carrera por su vocación hacia la docencia. Algo emblemático en el contexto del triunfo revolucionario, fue la creación de la Escuela de Cuadros, a esta escuela ingresaban y se formaban los cuadros políticos del Frente Sandinista de Liberación Nacional.

Por este motivo, la Universidad busca nuevas formas de organización para gestionar con mayor eficiencia los procesos de aprendizajes, y en el año de 1983 crea la Escuela de Geografía y la Escuela de Historia con sus respectivas carreras en la modalidad diurna y sabatina (Planes de Estudios 1982 de la Facultad de Ciencias de la Educación), y en el caso de la carrera Ciencias Sociales, esta no contaba con planta docente propia y queda dentro de ambas Escuelas, mismas que administraban su currículo y que le proporcionaban profesores de la especialidad para impartir las asignaturas de su Plan de Estudios en las modalidades diurno y nocturno. En esto cabe agregar que, de acuerdo a la revisión documental, la modalidad diurna tenía una duración de 4 años y la modalidad nocturna 5 años.

En este contexto histórico y con la vigencia de este Plan de Estudios que se extendió de 1982 a 1986, la Escuela de Geografía y la Escuela de Historia tuvieron un elevado auge, esto debido a que la demanda de las asignaturas de Ciencias Sociales creció porque a nivel curricular se estableció de manera obligatoria que todas las carreras de la UNAN-Managua debían incorporar en su Plan de estudios asignaturas de Ciencias Sociales, entre ellas Historia de la Revolución Popular Sandinista y Curso sobre la Problemática Actual, donde se estudiaban temas de carácter político-ideológico que venían a reforzar el proyecto revolucionario de Nicaragua. Y estas mismas características curriculares permeaban en la carrera Ciencias Sociales, puesto que en su Plan de Estudios 1982 se abordaban los temas políticos ideológicos en las asignaturas de Filosofía I y II, Historia de Nicaragua I, II, III y IV, Economía Política I y II e Historia del Movimiento Obrero y de Liberación Nacional.

\section{La Escuela de Ciencias Sociales (1987 - 1997)}

En el año 1987 las carreras de la UNAN-Managua inician un nuevo Plan de Estudios con fines de perfeccionamiento curricular, esto en el contexto de la agresión que el gobierno norteamericano llevaba a cabo contra Nicaragua a través de la guerra de la contrarrevolución.

Esta época también inicia con cambios significativos a nivel de la Facultad de Ciencias de la Educación, pues ocurre que las carreras de Geografía y de Historia entran en liquidez debido a su escaza demanda estudiantil, y se opta por crear la Escuela de Ciencias Sociales, la cual quedó regida de la siguiente manera: Dirección

6 Fuente: Entrevista a profesor Lobato 
de Escuela, Departamento de Geografía, Departamento de Historia y Departamento de Metodología de la Enseñanza y la Investigación (Lobato, 2020); para entonces, el personal docente adscrito a las antiguas Escuela de Geografía y Escuela de Historia, queda expresamente dedicado a impartir clases en la carrera Ciencias Sociales y a otras carreras que cursaban asignaturas de ciencias sociales.

Así, la Carrera Ciencias Sociales era coordinada por el Director de la Escuela de Ciencias Sociales, y en este año inicia un nuevo Plan de Estudios caracterizado por su énfasis en fortalecer la formación político-ideológica y coadyuvar al proyecto revolucionario del Frente Sandinista en Nicaragua, es por ello que se incorporaron asignaturas como Filosofía I y II, Historia de Nicaragua I, II, III y IV, Historia del Movimiento Obrero y de Liberación Nacional, Economía Política I y II, además, con frecuencia se solían realizar actividades curriculares y extracurriculares con enfoque político-ideológico que venían a reforzar el proyecto revolucionario nicaragüense, tales como apoyar en los cortes de café, algodón y participar en las jornadas rojinegras que organizaba la Universidad.

Para este periodo, la carrera Ciencias Sociales se ofertaba en el turno vespertino y nocturno, y en ambos era evidente una notable concurrencia de demanda estudiantil. Un factor incidente en este aspecto fue el programa de becas salarios que el Ministerio de Educación (MED) inauguró en 1989, esto con el objetivo de motivar a profesores a estudiar la carrera de Ciencias Sociales recibiendo su salario completo.

En cuanto a su Plan de Estudios propiamente dicho, las cohortes de 1987 a 1991 experimentaron otro enfoque en su formación profesional, habiéndose establecido la siguiente distribución académica de las 45 asignaturas que formaban parte del Plan de Estudios 1987: el 55.5\% estaba conformado por asignaturas disciplinares que correspondían al área de Geografía, Historia, Filosofía y Economía, el 15.5\% lo ocupaban las asignaturas básicas, el 13.6\% las asignaturas de corte psicopedagógico, solamente el $8.8 \%$ correspondía a las didácticas especiales, y apenas el 6.6\% correspondía a asignaturas propiamente de investigación.

Hacia el año de 1991 la Universidad planteó la necesidad de formar profesionales altamente calificados que dieran respuesta a las nuevas demandas que el contexto nacional imponía, en este caso, el inicio de los 16 años neoliberales en Nicaragua. Por tal motivo, la Facultad de Ciencias de la Educación organizó y desarrolló perfiles orientados hacia las disciplinas humanistas (Lobato et al, 2005), y es la Escuela de Ciencias Sociales a través de los Departamentos de Geografía e Historia, quienes presentan ante el Consejo Universitario la carrera de Geografía y la carrera de Historia, con el objetivo de formar profesionales en esta área del conocimiento, alejándose así del campo educativo propiamente. A partir de este momento, la Escuela de Ciencias Sociales empieza a administrar tres carreras: Ciencias Sociales, Geografía e Historia. 
El Plan de Estudios 91 se desarrolló sirviéndose en los turnos regular y sabatino, y para este entonces la demanda estudiantil se caracterizó por ser significativa. Asimismo, en este Plan de Estudios, en su modalidad sabatina, se instituyó la Modalidad de Graduación de Profesor en Educación Media (PEM), a quienes aprobaban los primeros seis semestres de su Plan de Estudios.

Con el Plan de Estudio 1991 los estudiantes de la carrera de Ciencias Sociales estudiaban tres años para obtener el título de Profesor de Educación Media en ciencias sociales y después para terminar su licenciatura ellos decidían en que mención querían terminar sus estudios y elegían entre Geografía e Historia. Es decir, bifurcaban, unos se especializaban en educación con mención en Geografía o Historia. Otro acontecimiento de este periodo es que entre los años de 1993 a 1994, es que la Facultad de Ciencias de la Educación pasa a llamarse Facultad de Ciencias de la Educación y Humanidades, en este caso, la carrera pasa a formar parte de esta Facultad.

\section{Coordinación adscrita al decanato (1998 - 2019)}

En el año 1997 en la UNAN-Managua se crea la departamentalización, es decir, se crean los Departamentos académicos y desaparece la anterior estructura organizativa por Escuelas. Así, se conforma el Departamento de Geografía y el Departamento de Historia, ambos con sus respectivo director, secretaria, colectivo docente y colectivos de asignaturas; Geografía quedó ubicada en el pabellón 44 e Historia en el pabellón 18, ambos formando parte de la Facultad de Ciencias de la Educación y Humanidades.

En este entonces, la Normativa de cada Departamento establecía que estas debían ofertar su respectiva carrera, y en el caso de la carrera Ciencias Sociales, esta de cierta manera queda huérfana, porque no pertenecía ni a Geografía, ni a Historia, ni a Filosofía, porque eran departamentos que ofertaban sus carreras como ciencias de carácter humanístico, sin contemplar el perfil educativo. Sin embargo, por afinidad al perfil profesional de la carrera Ciencias Sociales, esta quedó bajo la coordinación del profesor Freddy Montenegro Blanco quien era Licenciado en Ciencias de la Educación con mención en Ciencias Sociales y máster en Didáctica de las Ciencias Sociales.

Cabe precisar que en este tiempo la carrera Ciencias Sociales no tenía un local físico para funcionar como coordinación independiente, sino que esta la ejercía el profesor Freddy Montenegro desde su cubículo en el Departamento de Historia (Pabellón 18), mismo al cual el pertenecía como docente. Debido a esta directa influencia de quien coordinaba la carrera, la visión del currículo de Ciencias Sociales se centró en las asignaturas disciplinares, particularmente en las asignaturas del Departamento de Geografía y el Departamento de Historia, dejando marginadas aquellos componentes de carácter educativo. Como consecuencia, con la transformación curricular que la UNAN-Managua llevó a cabo en el año 1999, el plan de estudios quedó estructurado de la siguiente manera: 
- Tres asignaturas del área básica (7.5\%)

- Seis asignaturas del área psicopedagógica (15\%)

- Veintiún asignaturas del área disciplinar de Geografía, Historia, Economía y Filosofía (52.5\%)

- Una asignatura del área de didáctica especial (2.5\%)

- Tres asignaturas del área de investigación (7.5\%)

- Seis asignaturas del área socioeducativa (15\%)

Este Plan de Estudios (1999) también contemplaba el título de Profesor de Educación Media (PEM), manteniendo como único requisito la aprobación de los primeros ocho semestre del Plan de Estudios, asimismo, puede observarse la poca presencia de asignaturas de didáctica de la especialidad y la ausencia de prácticas profesionales en el campo educativo o social, por consiguiente, quedan retos y desafíos en los graduados de la carrera Ciencias Sociales, a esto hay que agregarle que este plan de estudios duró 13 años en su implementación (1999-2012).

En 1998 asume la coordinación de la carrera el profesor Freddy Montenegro Blanco, la ejercía desde el Departamento de Historia. En el año 2007 el maestro Freddy se jubila y asume la coordinación el decanato de la Facultad por un periodo de ocho meses. Luego asumí yo formalmente la coordinación de la carrera en el año 2008 (Orozco, 2021).

En el año 2012 se da la transformación curricular, la que se implementó a partir del año 2013, revisando así el plan de estudios de la carrera y se revisan los documentos curriculares del MINED para conocer en que asignaturas se tenían que modificar en cuanto a competencias y contenidos, formando a los estudiantes en la parte científica que les servirá en su práctica docente, entonces se libera carga horaria y esto nos permite tener un poco más de autonomía en la carrera.

Para el año 2013 la carrera de Ciencias sociales está bajo la coordinación del maestro Julio Orozco Alvarado, quien es Licenciado en Ciencias Sociales, Master en Didáctica de las Ciencias Sociales y Doctor en investigación e intervención social, la oficina de la coordinación de la carrera estaba ubicada en el pabellón 40. Como consecuencia, con la transformación curricular que la UNAN-Managua llevó a cabo en el año 2013, el plan de estudios quedó estructurado de la siguiente manera:

- Siete asignaturas del área básica (14.8\%)

- Cinco asignaturas del área psicopedagógica (10.6\%)

- Quince asignaturas del área disciplinar de Geografía, Historia, Economía y Filosofía (31.9\%)

- Tres asignaturas del área de didáctica especial (6.3\%)

- Tres asignaturas del área de investigación (6.3.5\%) 
- Once asignaturas del área socioeducativa (23.4\%)

- Tres asignaturas de Prácticas (6.3\%)

Este Plan de Estudios de 2013 también contemplaba el título de Profesor de Educación Media (PEM), manteniendo como requisito la aprobación de los primeros seis semestre del Plan de Estudios y la realización del Curso de Graduación (PEM), asimismo, ya se puede observar presencia de asignaturas de didáctica de la especialidad, aumenta el porcentaje en áreas socioeducativas y de prácticas profesionales en el campo educativo o social, por consiguiente, la carrera va aterrizando a un perfil constructivista de los graduados de la carrera Ciencias Sociales.

Para el año 2014 ya cuando retoma el gobierno el Frente Sandinista retornaron una cantidad de profesores a través del convenio MINED-UNAN-Managua a profesionalizarse, para erradicar el empirismo en las aulas de clase y así brindar una educación de calidad, y se tiene memoria que en la carrera de Ciencias Sociales se tenían en primer año 80 estudiantes, lamentablemente terminaron como 36 profesores.

En el año 2016 se realizó una adecuación curricular en la carrera de Ciencias Sociales, permitiendo liberar la carga académica para la carrera y validando las asignaturas que necesitaban los estudiantes para profesionalizarse, permitiendo que no hubiera un divorcio entre el plan de estudio y los conocimientos que los estudiantes necesitaban en su área labora o práctica docente, formando educadores innovadores y facilitadores de nuevos conocimientos.

Durante el proceso de adecuación curricular que la UNAN-Managua llevó a cabo en el año 2016, el plan de estudios quedó estructurado de la siguiente manera: Seis asignaturas del área básica (12.7\%), tres asignaturas del área psicopedagógica (10.6\%), catorce asignaturas del área disciplinar de Geografía, Historia, Economía y Filosofía (31.9\%), tres asignatura del área de didáctica especial (6.3\%), cinco asignaturas del área de investigación (6.3.5\%), trece asignaturas del área socioeducativa (23.4\%), tres asignaturas Prácticas (6.3\%)

En este Plan de Estudios (2016), se puede observar más asignaturas de didáctica de la especialidad, aumenta las asignaturas de las áreas socioeducativas y de prácticas profesionales en el campo educativo o social, por consiguiente, la carrera lleva un perfil por competencia de los graduados de la carrera Ciencias Sociales. La carrera de Ciencias Sociales siempre une sus esfuerzos por participar en las Jornadas Universitarias Científicas (JUDC) con hasta 12 trabajos o artículos científicos ganando primeros y segundos lugares, porque se incentiva al estudiantado a la investigación siendo esta una gran fortaleza. 


\section{Ciencias Sociales al Departamento Multidisciplinario (2019 - actualidad)}

Para el año 2018 la coordinación de la carrera de Ciencias Sociales pasa al pabellón 48 junto con las carreras Cultura y Artes, Danza, Educación Física y Deportes y Educación Comercial, junto con el colectivo de la asignatura Seminario de Formación Integral y se conforma el Departamento Multidisciplinarios dirigido por su director Dr. Julio Orozco Alvarado.

En el año 2020 la UNAN-Managua, inicia un proceso de perfeccionamiento curricular con enfoque por competencias, en el que la carrera Ciencias Sociales, de forma voluntaria entra en un proceso de pilotaje, trabajando de forma integrada todos los componentes curriculares y con Bases Orientadoras de la Acción (BOA), donde los profesores planifican la docencia y la evaluación de forma integrada. En el proceso de transformación curricular se trabajó en equipo tanto docentes horarios, docentes de plantas y el director del Departamento.

En el proceso de transformación curricular para el año 2020 el plan de estudio por competencia está organizado por ejes disciplinar y estos se desglosan en componentes curriculares, quedando de la siguiente forma:

- Eje disciplinar de Comunicación: Dos componentes curriculares

- Eje disciplinar Informática: un componente curricular

- Eje disciplinar Económico y social: Cuatro componentes curriculares

- Eje disciplinar Histórico: Cinco componentes curriculares

- Eje disciplinar Geográfico: Cuatro componentes curriculares

- Eje disciplinar de Formación docente: Seis componentes curriculares

- Eje disciplinar Promotoría educativa: Dos componentes curriculares

- Eje disciplinar Didáctica Especial: Dos componentes curriculares

- Eje disciplinar de Investigación: Tres componentes curriculares

- Ejes Optativos: Seis componentes curriculares

- Ejes Electivos: cuatro componentes curriculares

- Ejes disciplinar Integrador: Diez componentes curriculares

En el programa de estudio por competencia los ejes disciplinar optativos contemplan contenidos emergentes de la educación y de las Ciencias Sociales con la finalidad de abordar las teorías y tendencias actuales de esta área del conocimiento, de manera que permita al futuro profesional ejercer de manera coherente y de acuerdo a los conocimientos actualizados de su área del conocimiento.

De acuerdo a los ejes disciplinar electivos estas las pueden cursar los profesionalizante en cualesquier carreras que oferte la Universidad, esto con la finalidad de fortalecer su formación profesional y humanística. Los estudiantes podrán cursar 
los componentes curriculares electivos durante sus cinco años de estudio de la carrera y los componentes integradores estos permiten un aprendizaje y vínculo entre universidad-sociedad evidenciándose las competencias alcanzadas por el estudiante, siendo necesario implementar estrategias de aprendizaje integradoras, para generar aprendizajes significativos.

Para el año 2020 la carrera de Ciencias Sociales da apertura un 25 de agosto a la I cohorte de la Maestría en Ciencias de la Educación con énfasis en Didáctica de las Ciencias Sociales con una matrícula de 16 maestrandos, esta se imparte de manera presencial en el turno dominical.

En el 2020 se realizó una sistematización como carrera del pilotaje para dejar evidencia del arduo trabajo que se estaba realizando y así mejorar en los próximos años. Se continúa avanzando pensamos en abrir la II cohorte de la maestría Ciencias de la Educación con énfasis en Didáctica de las Ciencias Sociales, hemos tenido también avances significativos, se ha ido ampliando la planta docente de la carrera de Ciencias Sociales ahora somos 8 docentes horarios y 3 de planta.

\begin{tabular}{|c|c|c|c|c|c|c|}
\hline 1960 & 1980 & 1982 & $1983-1986$ & 1987 & 1991 & 1993-1994 \\
\hline $\begin{array}{l}\text { El } 7 \text { de julio de } \\
\text { 1960, se crea la } \\
\text { carrera CC.SS } \\
\text { como una sede } \\
\text { de la UNAN- } \\
\text { León }\end{array}$ & $\begin{array}{l}\text { Alta demanda } \\
\text { universitaria } \\
\text { por el triunfo } \\
\text { de la R.P.S. }\end{array}$ & $\begin{array}{l}\text { Separación UNAN- } \\
\text { Managua y UNAN- } \\
\text { León por decreto } \\
1036 \text { del } 29 \text { de } \\
\text { abril de } 1982\end{array}$ & $\begin{array}{l}\text { Se crea la } \\
\text { Escuela de } \\
\text { Ciencias } \\
\text { Sociales. }\end{array}$ & $\begin{array}{l}\text { Transformación } \\
\text { curricular de la } \\
\text { UNAN-Managua }\end{array}$ & $\begin{array}{l}\text { Reforma curricular } \\
\text { con énfasis en } \\
\text { Geografia e } \\
\text { Historia. }\end{array}$ & $\begin{array}{c}\text { Nace la Facultad } \\
\text { de Ciencias de la } \\
\text { Educación y } \\
\text { Humanidades }\end{array}$ \\
\hline
\end{tabular}

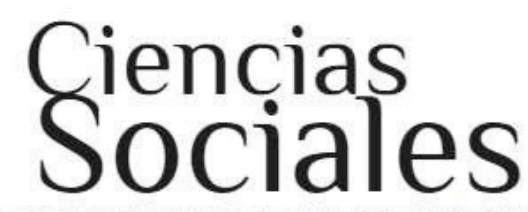

Formando profesionales competentes para el desarrollo del pais en los ámbitos educativos, investigativos y sociales.

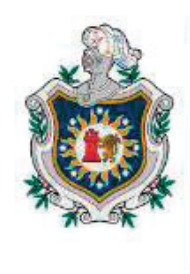

UNIVERSIDAD NACIONAL AUTÓNOMA DE NICARAGUA, MANAGUA

UNAN-MANAGUA

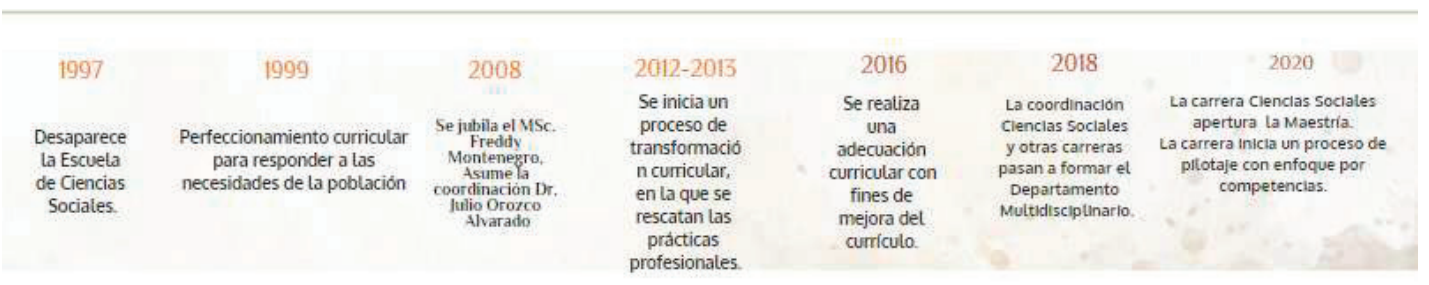

Figura 1: Línea de tiempo carrera Ciencias Sociales 


\section{Discusión y conclusiones}

A través de la realización de entrevistas estructuradas se recolectaron testimonios de vidas que permitió conocer acerca del origen y fundación de la carrera Ciencias Sociales de la Universidad Nacional Autónoma de Nicaragua, Managua (UNANManagua). Los testimonios facilitaron indagar sobre el área académica y administrativa que históricamente ha caracterizado a la carrera Ciencias sociales desde su surgimiento como Escuela, después la Departamentalización, luego queda bajo coordinación hasta culminar en el Departamento Multidisciplinario.

La carrera de Ciencias Sociales siempre ha tenido bien claro el perfil de sus egresados a lo largo de su devenir histórico que es formar profesionales en Educación que enseñen las diferentes asignaturas que la conforman en Institutos de Educación Secundaria y Centros de Educación Técnica del país, este profesional con carácter humanista capaz de comunicarse en diferentes contextos y que sea un investigador de carácter socioeducativo y que posee habilidades para brindar acompañamiento pedagógico y asesoría escolar.

También es importante resaltar que la carrera desde su inicio en los años 60 hasta la actualidad ha mantenido su demanda estudiantil, ya que se ha ofertado de acuerdo al contexto histórico nacional en modalidad nocturna, modalidad vespertina y la modalidad sabatina, y no ha dejado de ofertarse debido a que en el campo laboral se necesitan especialista que impartan las Ciencias Sociales en colegios de Educación Secundaria.

Para ir dando respuesta a la transformaciones y avances científicos-tecnológico la carrera Ciencias Sociales ha tenido varias transformaciones curriculares con el objetivo de preparar a profesionales que lleven a la práctica lo aprendido y que no exista un divorcio entre el plan de estudio de la carrera y el plan de estudio del Ministerio de Educación, esto permite una armonización entre el currículo, actualmente el currículo de la carrera es por competencia.

\section{Lista de referencia}

Bernal, C. (2010). Metodología de la Investigación. (3era edición). Pearson Educación: Colombia.

Casarini, M. (1999). Teoría y diseño curricular. México. Trillas.

Genet, Alejandro. (2020, 12 de noviembre) Entrevistas de A, Díaz y R, Dávila. [Cinta de audio]. Proyecto Historia de la carrera Ciencias Sociales. Managua, Nicaragua. 
Hernández Sampieri, R., Fernández Collado, C. y Baptista Lucio, P. (2014). Metodología de la Investigación (6ta Edición). Distrito Federal, México: McGraw-Hil: Interamericana Editores

Julia. (2020, 28 de octubre) Entrevistas de A, Díaz y R, Dávila. [Cinta de audio]. Proyecto Historia de la carrera Ciencias Sociales. Managua, Nicaragua.

Lobato, Alfredo. (2020, 01 de noviembre) Entrevistas de O, Julio y R, Dávila. [Cinta de audio]. Proyecto Historia de la carrera Ciencias Sociales. Managua, Nicaragua.

Lobato, L. (2005). Haciendo historia: Reseña del Departamento de Historia de la UNANManagua. UNAN.

Morazán, Francisco (2015). Perfil académico profesional de egreso. Universidad Pedagógica Nacional Recuperado de https://web.upnfm.edu.hn/tecnicasps/index.php/ ct-menu-item-10

Orozco, Julio. (2021, 27 de enero) Entrevista de C, Herrera. [Cinta de audio]. Proyecto Historia de la carrera Ciencias Sociales. Managua, Nicaragua.

Rodríguez, A., Luque, R. y Navas, A. (2014, junio). Usos y beneficios de la historia oral. Reidocrea, (3):193-200. Recuperado el 30 de enero de 2021, de https://digibug.ugr.es/bitstream/handle/10481/32326/ReiDoCrea3A24. pdf? sequence $=6$ \&isAllowed $=\mathrm{y}$

UNAN-Managua. (2019). 37 años de la UNAN-Managua como institución independiente. Recuperado el 22 de febrero de 2021 de https://cira.unan.edu.ni/index. $\mathrm{php} / 37$-anos-de-la-unan-managua-como-institucion-independiente/

UNAN-Managua. (1982). Plan de Estudios 1982 de la carrera Ciencias Sociales. Managua, Nicaragua: AUTOR.

UNAN-Managua. (1987). Plan de Estudios 1987 de la carrera Ciencias Sociales. Managua, Nicaragua: AUTOR.

UNAN-Managua. (1999). Plan de Estudios 1999 de la carrera Ciencias Sociales. Managua, Nicaragua: AUTOR.

UNAN-Managua. (2013). Plan de Estudios 2013 de la carrera Ciencias Sociales. Managua, Nicaragua: AUTOR.

UNAN-Managua. (2016). Plan de Estudios 2016 de la carrera Ciencias Sociales. Managua, Nicaragua: AUTOR. 